TITLE:

\title{
Spatial population distribution of laser ablation species determined by self-reversed emission line profile
}

\author{
$\operatorname{AUTHOR}(\mathrm{S}):$
}

Sakka, T; Nakajima, T; Ogata, YH

\section{CITATION:}

Sakka, T ... [et al]. Spatial population distribution of laser ablation species determined by self-reversed emission line profile. JOURNAL OF APPLIED PHYSICS 2002, 92(5): 2296-2303

\section{ISSUE DATE:}

2002-09-01

URL:

http://hdl.handle.net/2433/50401

\section{RIGHT:}

Copyright 2002 American Institute of Physics. This article may be downloaded for personal use only. Any other use requires prior permission of the author and the American Institute of Physics. 


\title{
Spatial population distribution of laser ablation species determined by self-reversed emission line profile
}

\author{
Tetsuo Sakka, ${ }^{\text {a) }}$ Takashi Nakajima, and Yukio H. Ogata \\ Institute of Advanced Energy, Kyoto University, Uji, Kyoto 611-0011, Japan
}

(Received 4 February 2002; accepted for publication 22 June 2002)

\begin{abstract}
We propose a method for determining the spatial distribution of population densities for the species in laser-produced plasma. Our method relies on the parameter fittings of the experimentally observed self-reversed emission profiles to the model which is based on the calculation of one-dimensional radiative transfer. Employed parameters in the model represent spatial distribution of emitters, absorbers, and plasma free electrons. Since the density of plasma electrons has a spatial dependence, Stark shifts and broadenings are incorporated in a position-sensitive manner. After a general description of the method, we have specifically applied it to the laser-ablated Al plasma, where $\mathrm{Al}(\mathrm{I}){ }^{2} P^{\circ}-{ }^{2} S$ emission line is employed for the analysis. In this specific example, we find that the accuracy of the fittings is significantly improved due to the presence of two emission lines originating from the fine structure, i. e., ${ }^{2} P_{1 / 2}^{\circ}{ }^{2} S_{1 / 2}$ and ${ }^{2} P_{3 / 2}^{\circ}-{ }^{2} S_{1 / 2}$. In particular, the depth of the self-reversed structure turns out to be very sensitive to the position-dependent upper and lower level populations, which enables us to accurately determine the spatial variation of the laser-ablated species in these states. Furthermore, the calculated profile is almost unchanged with temperatures employed for fittings. This means that the present method gives reliable values of the parameters for the spatial distributions, even if the temperature is not precisely known. (C) 2002 American Institute of Physics. [DOI: 10.1063/1.1500419]
\end{abstract}

\section{INTRODUCTION}

Laser ablation is widely applied to material processing, such as thin-film deposition, cluster formation, chemical reactions, and surface modifications. ${ }^{1-3}$ To improve the quality of the resultant materials, a sound understanding of the plume dynamics and physical and chemical properties of the ablated species is required. In addition, ablation plume analysis is important for the surface elemental analysis based on laser-induced breakdown spectroscopy. ${ }^{4,5}$ Among several methods of plume analysis, optical spectroscopy ${ }^{6-8}$ has good temporal and spatial resolution. In order to extract desired information from the emission spectra, it is necessary to establish reliable procedures for quantitative analysis of the spectra.

However, the line profile observed in the emission spectra of the laser ablation plume is distorted by several factors, such as (1) a high optical thickness at an atomic transition frequency (opacity effect), (2) Stark shifts and broadening due to a comparatively high electron density of the plasma, and its inhomogeneous spatial distribution, and (3) different spatial distributions of the population for the upper and lower levels involved in the transition. The distortion mechanisms (1) and (3) are the direct consequence of self-absorption.

It has been known that the profile of an optically thick line can not be well described without the self-absorption effects, especially when a self-reversed structure appears in a line profile. The mechanism of self-reversal is reviewed in the articles by Cowan and Dieke, ${ }^{9}$ and also by Zwicker. ${ }^{10}$

\footnotetext{
a) Author to whom correspondence should be addressed; electronic mail: t-sakka@iae.kyoto-u.ac.jp
}

The analytical formulation developed in the early studies is still used in a recent work ${ }^{11}$ to understand the effects of spatial inhomogeneity of emitting and absorbing species. On the other hand, a numerical analysis has also been compared with the analytical model. ${ }^{12,13}$ The self-reversed line profile has been analyzed for the $\mathrm{x}$ ray, ${ }^{14-17}$ visible, ${ }^{18}$ and infrared emissions ${ }^{19}$ of laser-produced plasma, discharge lamps, and heated materials, respectively. Under typical conditions for laser nuclear fusion experiments, a self-reversed line appears in the x-ray region. On the contrary, for laser fluences of $100 \mathrm{~mJ} \mathrm{~cm}^{-2}$ to $100 \mathrm{~J} \mathrm{~cm}^{-2}$ at which most experiments of material processings by laser ablation are carried out, the self-reversed line appears in the UV/visible region. However, the studies on self-reversed spectral lines of laser-produced plasma for the laser fluence in this range, and therefore in the UV/visible region, are rather limited. Using a two-zone model, Hermann et al. ${ }^{20}$ have carried out spatially-as well as temporally-resolved study of the self-reversed line of Ti(II) in a nitrogen gas atmosphere. In the two-zone model, radiative transfer through both zones, the central and peripheral regions, was calculated, assuming that each zone is characterized by a uniform temperature. A similar analysis, by dividing the emission region into two subregions with different temperatures, had been performed in the case of $\mathrm{dc}$ arc plasma. ${ }^{21}$ Analysis of self-reversed line profiles for laser ablation plasma beyond the two-zone model has not yet been carried out. Whether the two-zone model is sufficient or not would depend on the experimental conditions, and needs to be examined by detailed analysis using a continuous spatial distribution function for the ablated species as emitters and absorbers. Although the spatial behavior of the plasma pa- 
rameters for the laser ablation plume has been drawing considerable attention, ${ }^{22,23}$ very few studies on the analysis of self-reversed spectra have been performed in order to determine the spatial distribution of laser ablation species.

In the present article, we propose a model to account for the self-reversed line profile, by which the spatial distribution of population densities for the species in the laserproduced plasma can be obtained. Our model goes beyond the two-zone treatment and is based on the assumption that the emitting and absorbing atoms are continuously distributed in the plasma region. Also, the effects of positiondependent Stark shift and Stark broadening due to the spatially distributed plasma electrons are taken into account. The parameters to describe the spatial distribution of the emitters, absorbers, and plasma electrons are determined either through the fitting process, or independently from the model. Numerical calculations are carried out to synthesize a line profile, and applied to the ${ }^{2} P^{\circ}-{ }^{2} S$ transition of $\mathrm{Al}(\mathrm{I})$. This transition has a fine structure, giving rise to two lines at $394.401 \mathrm{~nm}\left({ }^{2} P_{1 / 2}^{\circ}-{ }^{2} S_{1 / 2}\right)$ and $396.152 \mathrm{~nm}\left({ }^{2} P_{3 / 2}^{\circ}-{ }^{2} S_{1 / 2}\right)$. Both lines can be simultaneously calculated by using a common set of parameters, and therefore, the parameters obtained through the fitting process should improve their reliability.

\section{MODEL}

The self-reversed line profile can be intuitively understood as follows; the broadened emission spectrum from the central region of the plume is absorbed by the lower level atoms in an optically thin peripheral region. Furthermore, the self-reversed emission line sometimes shows an asymmetric profile, which cannot be explained only in terms of the selfabsorption mechanism due to the optical thickness. A mechanism which causes a spectral line shift must exist. Among the mechanisms causing a shift of emission lines, we assume that the Stark effect plays a dominant role in the case of laser-produced plasma; it can be asymmetric as observed in the actual spectral lines, and the electron density in the plume can be sufficiently high to result in considerable broadening and shift. Therefore, the plasma electron density, which is closely related to the Stark effect, and its spatial distribution with respect to the population distribution of emitting and absorbing atoms must be the important factors. The essence of the model, which is based on the calculation of the radiative transfer in plasma, is that the spatial distribution of plasma electron density, and the population densities of atoms in the upper and lower states can be varied independently from each other.

The spectral radiant flux density $I(x, \nu)$ $\left(\mathrm{J} \mathrm{m}^{-2} \mathrm{~s}^{-1} \mathrm{~Hz}^{-1} \mathrm{sr}^{-1}\right)$, defined as a function of the radiation frequency $\nu$ and the distance $x$ along the line of the observation, follows a one-dimensional radiative transfer equation ${ }^{24}$ given by

$$
d I(x, \nu)=[\epsilon(x, \nu)-\kappa(x, \nu) I(x, \nu)] d x,
$$

where $\epsilon(x, \nu)\left(\mathrm{J} \mathrm{m}^{-3} \mathrm{~s}^{-1} \mathrm{~Hz}^{-1} \mathrm{sr}^{-1}\right)$ is the spectral emission coefficient measured per unit volume per unit solid angle, and $\kappa(x, \nu)\left(\mathrm{m}^{-1}\right)$ is an absorption coefficient. The use of
Eq. (1) assumes that all the optical paths of the collected light are characterized by the same emission and absorption profile. By integrating Eq. (1) over $x$ which is sufficiently long compared with the length in which emission and absorption take place, we obtain the radiant flux density at the far field, $I(\nu)$, i. e.,

$$
I(\nu)=\frac{1}{\phi(\infty, \nu)} \int_{-\infty}^{\infty} \phi(x, \nu) \epsilon(x, \nu) d x,
$$

where

$$
\phi(x, \nu)=\exp \left[\int \kappa(x, \nu) d x\right] .
$$

In order to estimate $I(\nu)$, we need $\epsilon(x, \nu)$ and $\kappa(x, \nu)$ as a function of $x$. In terms of the Einstein coefficients, $A, B_{21}$, and $B_{12}$, which represent spontaneous emission, induced emission, and absorption, respectively, they are given $\operatorname{as}^{24,25}$

$$
\begin{aligned}
& \epsilon(x, \nu)=\frac{A n_{2}(x) h \nu f(x, \nu)}{4 \pi}, \\
& \kappa(x, \nu)=\frac{\left[B_{12} n_{1}(x)-B_{21} n_{2}(x)\right] h \nu f(x, \nu)}{c},
\end{aligned}
$$

with $n_{1}(x)$ and $n_{2}(x)$ being the population densities of the lower and upper levels, respectively, $h$ is the Planck constant, and $c$ is the light velocity. As is well known, $B_{12}$ and $B_{21}$ are connected through $B_{12}=\left(g_{2} / g_{1}\right) B_{21}$, where $g_{1}$ and $g_{2}$ are the degeneracies of the lower and upper levels, respectively. The function $f(x, \nu)$ gives the spectral distribution of atomic transition for a given $x$, where $\nu$ is the radiation frequency. This function is normalized as

$$
\int_{-\infty}^{\infty} f(x, \nu) d \nu=1 .
$$

Note that the spectral distribution function is not simply a function of $\nu$, but also a function of $x$. According to the theory of Stark shifts and Stark broadenings for neutral and singly ionized emitters, ${ }^{26,27}$ the full width at half maxima $\Delta \lambda_{\text {width }}$, and the shift $\Delta \lambda_{\text {shift }}$, are described as a function of the electron density $n_{e}\left(\mathrm{~m}^{-3}\right)$, i. e.,

$$
\begin{aligned}
\Delta \lambda_{\text {width }}= & 2 W\left(\frac{n_{e}}{10^{22}}\right)\left[1+1.75 \alpha\left(\frac{n_{e}}{10^{22}}\right)^{1 / 4}\left(1-0.75 n_{D}^{-1 / 3}\right)\right], \\
\Delta \lambda_{\text {shift }}= & D\left(\frac{n_{e}}{10^{22}}\right) \pm 2.0 \alpha\left(\frac{n_{e}}{10^{22}}\right)^{1 / 4}\left(1-0.75 n_{D}^{-1 / 3}\right) \\
& \times W\left(\frac{n_{e}}{10^{22}}\right),
\end{aligned}
$$

where $W, D$, and $\alpha$, are the electron impact parameters for the Stark width, Stark shift, and the ion correction parameter, respectively. These parameters are independent of the electron density $n_{e}$, and are slowly varying functions of the electron temperature. The parameter $n_{D}$ represents the number of particles in the Debye sphere, the radius of which (Debye radius) is characterized by the screening of the ion charge. The first terms on the right-hand side of Eqs. (7) and (8) are due to the electron impact contribution, and the second terms 
are for the ion correction. Since the dependence of the second terms on the electron density is not linear, the width and the shift are not the linear functions of electron density, either, in general. However, the experimentally determined Stark width and shift parameters given in Ref. 28 are the coefficients for the linear functions of electron density, which give the best width and shift at the electron density employed in their measurement. This means that the nonlinear functions $\Delta \lambda_{\text {width }}$ and $\Delta \lambda_{\text {shift }}$ can be approximated as linear functions within a certain range of the electron density

$$
\begin{aligned}
& \Delta \lambda_{\text {width }}(x)=w n_{e}(x), \\
& \Delta \lambda_{\text {shift }}(x)=d n_{e}(x),
\end{aligned}
$$

where $w$ and $d$ are the experimentally determined Stark width and the Stark shift parameters, respectively. By using $\alpha=0.039$ and $D / W=1.554$, which are the theoretical values for the ${ }^{2} P^{\circ}-{ }^{2} S$ transition of $\mathrm{Al}(\mathrm{I})$ at $10000 \mathrm{~K},{ }^{26}$ and assuming that the experimental parameter $w$ is determined at the electron density of $1 \times 10^{23} \mathrm{~m}^{-3}$, the deviation from the linearity was found to be $19 \%$ for the width and $13 \%$ for the shift at the electron density of $1 \times 10^{25} \mathrm{~m}^{-3}$. This implies that the linear approximations given by Eqs. (9) and (10) are good approximations for the plasma with the electron density up to two or three orders of magnitude higher than the density at which the parameters $w$ and $d$ are determined. Therefore, we assume a Lorentzian profile with the width and shift given in Eqs. (9) and (10) from now on. This leads to the following position-dependent spectral distribution function

$$
f(x, \nu)=\frac{1}{\pi} \frac{\Delta \nu_{\text {width }}(x) / 2}{\left[\Delta \nu_{\text {width }}(x) / 2\right]^{2}+\left[\nu-\nu_{0}+\Delta \nu_{\text {shift }}(x)\right]^{2}},
$$

where $\nu_{0}$ is the unperturbed resonant frequency, $\Delta \nu_{\text {width }}(x)$ $=\left(c / \lambda_{0}^{2}\right) \Delta \lambda_{\text {width }}(x), \quad$ and $\quad \Delta \nu_{\text {shift }}(x)=\left(c / \lambda_{0}^{2}\right) \Delta \lambda_{\text {shift }}(x)$. Since the Stark parameters $w$ and $d$ are slowly varying function of temperature, we ignore their temperature dependence throughout the calculations.

The parameters described so far are those for the atomic transitions, i. e., $A, B_{21}$, and $B_{12}$, and those for the distortion of the spectral distribution function, namely, $w$ and $d$. Once the spatial distributions of the plasma electrons $n_{e}(x)$, the upper level population $n_{2}(x)$, and the lower level population $n_{1}(x)$ are given, the emission intensity $I(\nu)$ can be calculated by using Eq. (2) for each $\nu$. In order to compare the calculated $I(\nu)$ to the observed spectra, the convolution with the instrumental function, $g(\nu)$, should be performed, i.e.,

$$
I_{\text {calc }}(\nu)=\int_{-\infty}^{\infty} I(\mu) g(\nu-\mu) d \mu .
$$

The instrumental function is assumed to be a Gaussian distribution with a half width corresponding to the resolution of the whole detection system. If the detection efficiency is incorporated in $g(\nu), I_{\text {calc }}(\nu)$ should give a measured intensity.

In the remaining part of this section, we describe the spatial distribution functions to be used in the calculations. The plume produced by laser ablation is often found to be cylindrically symmetric along the axis normal to the target surface. In the case of a graphite target in air, the intensity profile for the lateral image of the plume is approximated by the Gaussian distribution, if the time delay from the ablation pulse is sufficiently long. ${ }^{29}$ At earlier times after the ablation pulse, the measured intensity distribution deviates from the Gaussian distribution. This is explained by the selfabsorption effect caused by the high density of emitters and absorbers, and does not mean that the spatial distribution of the emitters is not Gaussian. As long as the intensity profile for the low plasma density is well described by the Gaussian distribution, the spatial distribution of the electrons and atoms involved in the emission process may be well approximated by the Gaussian functions.

Based on the arguments given herein, we assume that $n_{e}(x), n_{2}(x)$, and $n_{1}(x)$ have Gaussian distributions with a center of distribution being the identical point. By letting the point at $x=0$ be the center of the plume, we obtain following distribution functions,

$$
\begin{aligned}
& n_{e}(x)=N_{e 0} \exp \left(-\frac{x^{2}}{\sigma_{e}^{2}}\right), \\
& n_{1}(x)=N_{10} \exp \left(-\frac{x^{2}}{\sigma_{1}^{2}}\right), \\
& n_{2}(x)=N_{20} \exp \left(-\frac{x^{2}}{\sigma_{2}^{2}}\right),
\end{aligned}
$$

where $\sigma_{e}, \sigma_{1}$, and $\sigma_{2}$ are the width parameters for the electrons and the atoms in the lower and upper levels, respectively, and $N_{e 0}, N_{10}$, and $N_{20}$ are their maximum densities at $x=0$, respectively. These six parameters are basically independent, although in actual calculations some restrictions will be imposed to reduce the number of adjusting parameters. The different distribution widths for the upper and lower levels, i. e., $\sigma_{1} \neq \sigma_{2}$, mean that the temperature gradually changes with the position $x$.

\section{APPLICATION TO THE ${ }^{2} \boldsymbol{P}^{\circ}{ }^{2} \boldsymbol{S}$ LINE OF AI(I)}

In this section, we apply the model to the emission line of $3 s^{2} 3 p^{2} P^{\circ}-3 s^{2} 4 s^{2} S$ transition of $\mathrm{Al}(\mathrm{I})$ in a laserproduced plasma. The lower state has a configuration of the ground state, i. e., (Ne core) $+3 s^{2} 3 p$. This configuration has a fine structure, i. e., a doublet, ${ }^{2} P_{3 / 2}^{\circ}$, and ${ }^{2} P_{1 / 2}^{\circ}$, with a splitting of $112 \mathrm{~cm}^{-1}$. Therefore, two transition lines appear, at $396.152 \mathrm{~nm}\left({ }^{2} P_{3 / 2}^{\circ}-{ }^{2} S_{1 / 2}\right)$ and $394.401 \mathrm{~nm}\left({ }^{2} P_{1 / 2}^{\circ}{ }^{2} S_{1 / 2}\right)$. We introduce suffices " $a$ " and " $b$ " for ${ }^{2} P_{3 / 2}^{\circ}$ and ${ }^{2} P_{1 / 2}^{\circ}$, respectively, for the parameters, if needed to be distinguished between each other.

The experimental setup for the spectrum measurement is very similar to that in our previous work. ${ }^{30} \mathrm{~A} 30 \mathrm{~mJ}$ pulse of the $1064 \mathrm{~nm} \mathrm{Nd:YAG} \mathrm{laser} \mathrm{(20} \mathrm{ns} \mathrm{pulse} \mathrm{duration)} \mathrm{was} \mathrm{fo-}$ cused onto an Al metal target $(99.999 \%$, Nilaco Co.) in atmospheric air by using a $10 \mathrm{~cm}$ focal-length lens. The lateral emission from the ablation plume was focused onto the entrance slit of a $1 \mathrm{~m}$ focal-length double dispersion spectrograph (Ritsu Oyo Kogaku, MC100N) equipped with two 1800 grooves/mm diffraction gratings. The slit width was set 
to $20 \mu \mathrm{m}$. An intensified charge coupled device (ICCD) (Princeton Instruments, ICCD-1024MTDGE/1) was used as a detector. The spectral resolution of this system is limited by the correlation between the adjacent pixels of the ICCD detector, which was $\sim 0.04 \mathrm{~nm}$. The duration of the time gate to drive the ICCD detector was $20 \mathrm{~ns}$ and the time delay from the ablation laser pulse was set to $1000 \mathrm{~ns}$. This time delay was employed, simply because at around this time scale, the spectrum is suitable for comparison with the present model, i. e., in very early stages $(<100 \mathrm{~ns})$, we have strong continuous spectral emission which gives an offset to the line spectra, while in the later stages ( $\gg 4000 \mathrm{~ns}$ ), the signal intensity becomes weak. It should be noted, however, that the model might be still applicable even at $<100 \mathrm{~ns}$, if the offset due to the continuous spectral emission were taken into account.

In order to simulate the spectra by using the present model, it is necessary to determine the parameters $\sigma_{e}, \sigma_{1}$, $\sigma_{2}, N_{e 0}, N_{10}$, and $N_{20}$. The estimation of these parameters is not very simple. Among them $\sigma_{e}$ is the $1 / e$ radius of the plasma and estimated from the image of the plume. Since our plume imaging experiments ${ }^{29}$ show that its full width at half maximum is $\sim 2 \times 10^{-3} \mathrm{~m}$, we assumed $\sigma_{e}=1.0 \times 10^{-3} \mathrm{~m}$ in the present analysis. The population distribution of the upper level should be very similar to the plasma distribution, because the plasma formation and the excitation to the upper state are expected to be closely related in a sense that both can originate from nonthermal processes. Therefore, we assume $\sigma_{2}=\sigma_{e}$. The parameter $\sigma_{1}$ is regarded as an adjustable parameter. As for the number densities, $N_{e 0}, N_{10}$, and $N_{20}$, we have very little experimental information. In some studies, the temperature of the plasma was estimated through the analysis of emission spectra, in which the relative populations in different energy states are obtained. The literature usually give very high temperatures of several thousands to more than ten thousand Kelvin. ${ }^{31-34}$ By assuming a temperature parameter $T_{0}$ to be the same order as the values in literature, the ratio $N_{20} / N_{10}$ can be estimated from the Boltzmann distribution, i. e.,

$$
\frac{N_{20}}{N_{10}}=\frac{g_{2}}{g_{1}} \exp \left(-\frac{h \nu_{0}}{k_{B} T_{0}}\right)
$$

where $k_{B}$ is the Boltzmann constant and $T_{0}$ is the temperature of $\mathrm{Al}$ atoms at the center of the light-emitting region. Note that the present model does not require the local thermal equilibrium. The use of the Boltzmann distribution and the introduction of the temperature parameter $T_{0}$ are solely for the purpose of estimating the ratio, $N_{20} / N_{10}$, by giving a reasonable value of $T_{0}$. In the present work, we assumed $T_{0}=4000 \mathrm{~K} .{ }^{34}$ We examined the effect of $T_{0}$ on the calculated spectral profile over the range of $2000<T_{0}$ $<20000 \mathrm{~K}$. Although the absolute intensity of the profile varies over six orders of magnitude, it turns out that the parameters obtained through the fitting process, except for the scaling factor, are hardly influenced by $T_{0}$. This means that the accuracy of the parameters determined through the fitting process is not influenced by the value of $T_{0}$. At the same time, if the absolute emission intensity were measured, the temperature could be obtained by comparing with the model.

As mentioned earlier, the ${ }^{2} P^{\circ}$ state has a fine-structure doublet, ${ }^{2} P_{3 / 2}^{\circ}$ and ${ }^{2} P_{1 / 2}^{\circ}$. Following Eq. (14), we introduce the spatial distribution of the populations for these levels, $n_{1 a}(x)$ and $n_{1 b}(x)$, respectively, as

$$
\begin{aligned}
& n_{1 a}(x)=N_{1 a} \exp \left(-\frac{x^{2}}{\sigma_{1 a}^{2}}\right), \\
& n_{1 b}(x)=N_{1 b} \exp \left(-\frac{x^{2}}{\sigma_{1 b}^{2}}\right),
\end{aligned}
$$

where $N_{1 a}$ and $N_{1 b}$ are the population density at the center of the plume for the levels, ${ }^{2} P_{3 / 2}^{\circ}$ and ${ }^{2} P_{1 / 2}^{\circ}$, respectively, and $\sigma_{1 a}$ and $\sigma_{1 b}$ are the distribution parameters. Since the energy difference between these levels $\left(112 \mathrm{~cm}^{-1}\right)$ is very small compared with the thermal energy, $\exp \left(-\Delta E_{a b} / k_{B} T\right) \simeq 1$ even at room temperature, where $\Delta E_{a b}$ is the energy difference between the fine structure levels. Therefore,

$$
\frac{n_{1 a}(x)}{n_{1 b}(x)}=\frac{N_{1 a} e^{-x^{2} / \sigma_{1 a}^{2}}}{N_{1 b} e^{-x^{2} / \sigma_{1 b}^{2}}}=\frac{g_{a}}{g_{b}},
$$

where $g_{a}$ and $g_{b}$ are the degeneracies of the doublet ${ }^{2} P_{3 / 2}^{\circ}$ and ${ }^{2} P_{1 / 2}^{\circ}$, respectively. From Eq. (19) it is obvious that

$$
\frac{N_{1 a}}{N_{1 b}}=\frac{g_{a}}{g_{b}},
$$

and

$$
\sigma_{1 a}=\sigma_{1 b}=\sigma_{1} .
$$

In the calculation, we regard $N_{1 b}$ as an adjustable parameter. The values of $N_{20}$ and $N_{1 a}$ are then determined by the relations, Eqs. (16) and (20), respectively. The other parameters, namely $A, B_{21}$, and $B_{12},{ }^{35}$ and two Stark parameters, $w$ and $d,{ }^{28}$ were taken from literature.

Summarizing this section, three parameters, $\sigma_{1}, N_{1 b}$, and $N_{e 0}$ are considered as adjustable parameters. By giving trial values to those parameters, we can calculate $n_{e}(x)$, $n_{1 a}(x), n_{1 b}(x)$, and $n_{2}(x)$, using Eqs. (13), (17), (18), and (15), as a function of the observation path $x$. Once $n_{e}(x)$ is thus determined, the Stark broadening and Stark shift are calculated as a function of $x$ by using Eqs. (9) and (10). Then the line profile is calculated from Eq. (11) as a function of $x$. After that, the emission and absorption coefficients are calculated from Eqs. (4) and (5), and the radiant flux density from Eq. (2). Finally, the spectrum convoluted with the instrumental function is calculated by Eq. (12). By varying the three adjustable parameters, $\sigma_{1}, N_{1 b}$, and $N_{e 0}$, we obtain the best fit to the experimental spectrum. Fitting was performed using a computer program which is based on iterative fitting to minimize the deviation of $I_{\text {calc }}(\nu)$ from the experimental spectra.

\section{DISCUSSION}

By adjusting the fitting parameters, the calculated spectrum reproduces the experimentally obtained self-reversed spectrum very well. In Fig. 1, an experimental spectrum is 


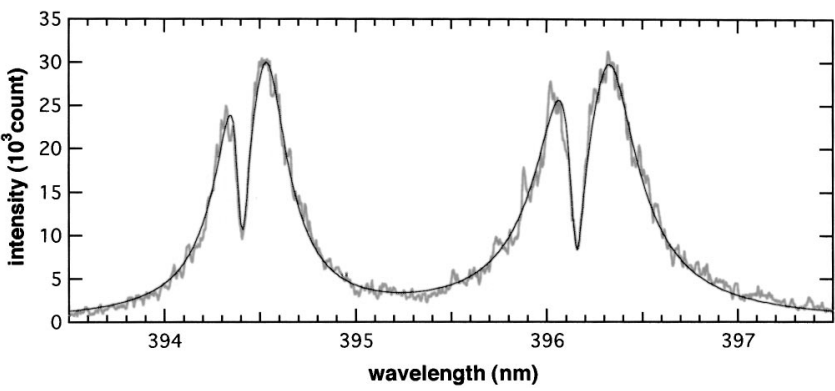

FIG. 1. Emission spectra obtained by the irradiation of pulsed Nd:YAG laser to an $\mathrm{Al}$ metal target in air. The time delay from the laser irradiation was set to $1000 \mathrm{~ns}$. The fit to the spectrum using the present model calculation is given by the solid line in the figure. The parameters used in the calculation are listed in Table I.

shown together with the best-fit spectrum. In order to examine the stability of the fitting, we repeated the fittings starting from one adjustable parameter to be ten times larger or smaller than the best-fit value. Except for the case starting from a larger $\sigma_{1}$, all the runs resulted in the convergence to the same best-fit values within $0.1 \%$. Although unreasonable initial values sometimes resulted in a nonconvergence, it has never resulted in a different set of the fitting parameters with a successful convergence. The best-fit parameters as well as all the other parameters are listed in Table I. The electron density, $N_{e 0}$, at the center of the plasma was obtained to be $3.11 \times 10^{23} \mathrm{~m}^{-3}$. This seems to be a reasonable value ${ }^{36}$ for the delay time of $1000 \mathrm{~ns}$ from the ablation pulse and the irradiation fluence employed in the present experiments. In order to reproduce a self-reversed line, the spatial distribution of the lower state represented by the parameter $\sigma_{1}$ has to be broader than that of the upper state $\sigma_{2}$; the light-emitting region necessarily has an envelope which is cooler than the

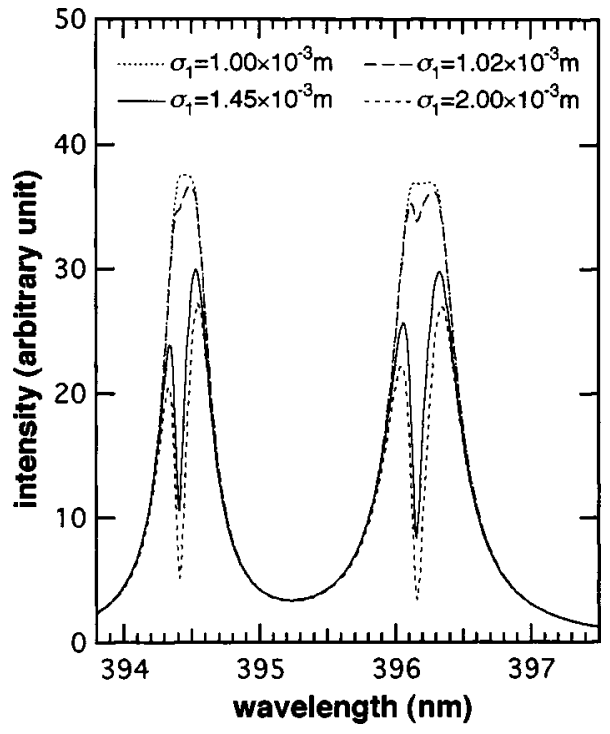

FIG. 2. Representative spectra obtained by the model calculation with various values of $\sigma_{1}$. All the other parameters were fixed to the values listed in Table I. Dotted line, long broken line, solid line, and short broken line represent $\sigma_{1}=1.00 \times 10^{-3}, 1.02 \times 10^{-3}, 1.45 \times 10^{-3}$, and $2.00 \times 10^{-3} \mathrm{~m}$, respectively.

central region. The value of $\sigma_{1}$ obtained by the fitting procedure was $1.45 \times 10^{-3} \mathrm{~m}$, with the parameter $\sigma_{2}$ fixed to $1.00 \times 10^{-3} \mathrm{~m}$.

The depth of the self-reversal was very sensitive to the ratio of $\sigma_{1}$ to $\sigma_{2}$. In Fig. 2, we plot a few representative spectra calculated with various values of $\sigma_{1}$ while keeping all the other parameters fixed to the values listed in Table I. As $\sigma_{1}$ increases, the self-reversal structure becomes more prominent, although the change in the spectral feature is limited only to the central or self-reversing part of the line pro-

TABLE I. Parameters used for the simulation of ${ }^{2} P^{\circ}-{ }^{2} S$ self-reversed spectra of $\mathrm{Al}(\mathrm{I})$.

\begin{tabular}{|c|c|c|c|}
\hline Symbol & Definition & Value (unit) & $\begin{array}{l}\text { Method of } \\
\text { evaluation }\end{array}$ \\
\hline$A_{2 a}$ & radiative rate for ${ }^{2} P_{3 / 2}^{\circ}-{ }^{2} S_{1 / 2}$ transition & $9.8 \times 10^{7}\left(\mathrm{~s}^{-1}\right)$ & Ref. 35 \\
\hline$A_{2 b}$ & radiative rate for ${ }^{2} P_{1 / 2}^{\circ}-{ }^{2} S_{1 / 2}$ transition & $4.93 \times 10^{7}\left(\mathrm{~s}^{-1}\right)$ & Ref. 35 \\
\hline$g_{a}$ & degeneracy of ${ }^{2} P_{3 / 2}^{\circ}$ level & 4 & Ref. 35 \\
\hline$g_{b}$ & degeneracy of ${ }^{2} P_{1 / 2}^{\circ}$ level & 2 & Ref. 35 \\
\hline$g_{2}$ & degeneracy of ${ }^{2} S_{1 / 2}$ level & 2 & Ref. 35 \\
\hline$\lambda_{a 0}$ & origin of ${ }^{2} P_{3 / 2}^{\circ}-{ }^{2} S_{1 / 2}$ transition & $396.152(\mathrm{~nm})$ & Ref. 35 \\
\hline$\lambda_{b 0}$ & origin of ${ }^{2} P_{1 / 2}^{\circ}{ }^{2} S_{1 / 2}$ transition & $394.401(\mathrm{~nm})$ & Ref. 35 \\
\hline$w$ & Stark width parameter & $4.22 \times 10^{-25}\left(\mathrm{~nm} / \mathrm{m}^{-3}\right)$ & Ref. 28 \\
\hline$d$ & Stark shift parameter & $2.42 \times 10^{-25}\left(\mathrm{~nm} / \mathrm{m}^{-3}\right)$ & Ref. 28 \\
\hline$T_{0}$ & temperature parameter & $4000(\mathrm{~K})$ & estimated $^{\mathrm{a}}$ \\
\hline$N_{e 0}$ & electron density parameter ${ }^{\mathrm{f}}$ & $3.11 \times 10^{23}\left(\mathrm{~m}^{-3}\right)$ & adjusted \\
\hline$N_{1 a}$ & population parameter of ${ }^{2} P_{3 / 2}^{\circ}$ state ${ }^{\mathrm{f}}$ & $1.23 \times 10^{22}\left(\mathrm{~m}^{-3}\right)$ & calculated $^{\mathrm{b}}$ \\
\hline$N_{1 b}$ & population parameter of ${ }^{2} P_{1 / 2}^{\circ}$ state ${ }^{\mathrm{f}}$ & $6.17 \times 10^{21}\left(\mathrm{~m}^{-3}\right)$ & adjusted \\
\hline$N_{20}$ & population parameter of ${ }^{2} S$ state $\mathrm{f}^{\mathrm{f}}$ & $6.68 \times 10^{17}\left(\mathrm{~m}^{-3}\right)$ & calculated $^{\mathrm{c}}$ \\
\hline$\sigma_{e}$ & distribution parameter of the electrons & $1.00 \times 10^{-3}(\mathrm{~m})$ & estimated $^{\mathrm{d}}$ \\
\hline$\sigma_{1}$ & distribution parameter of ${ }^{2} P^{\circ}$ state & $1.45 \times 10^{-3}(\mathrm{~m})$ & adjusted \\
\hline$\sigma_{2}$ & distribution parameter of ${ }^{2} S$ state & $1.00 \times 10^{-3}(\mathrm{~m})$ & assumed $^{\mathrm{e}}$ \\
\hline
\end{tabular}

${ }^{\mathrm{a}}$ Estimated from previous experiments.

${ }^{\mathrm{b}}$ Calculated so as to keep $N_{1 a} / N_{1 b}=g_{a} / g_{b}=2$.

${ }^{c}$ Calculated from $T_{0}$ and $N_{1 b}$ using Eq. (16).

${ }^{\mathrm{d} E s t i m a t e d ~ f r o m ~ o u r ~ m e a s u r e m e n t ~(R e f . ~ 29) . ~}$

${ }^{\mathrm{e}}$ Assumed to be the same as $\sigma_{e}$.

${ }^{\mathrm{f}}$ Corresponding to the population at the center of the plasma. 


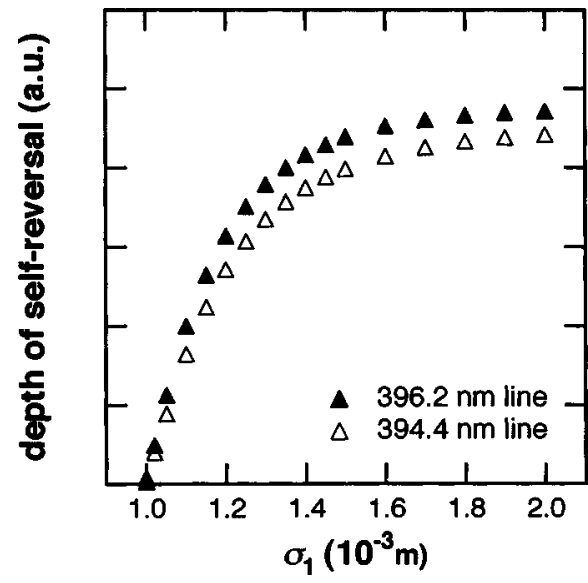

FIG. 3. The depth of the self-reversal in the spectra calculated by using various values of $\sigma_{1}$. The other parameters used in the calculation were fixed to the values listed in Table I. Solid triangles indicate the depth of the ${ }^{2} P_{3 / 2}^{\circ}{ }^{2} S_{1 / 2}(396.2 \mathrm{~nm})$ line while open triangles indicate that of the ${ }^{2} P_{1 / 2}^{\circ}-{ }^{2} S_{1 / 2}(394.4 \mathrm{~nm})$ line.

file. The wings of the lines are hardly affected by the values of $\sigma_{1}$. It should be noted that even a very small difference of $\sigma_{1}$ from $\sigma_{2}$ can give rise to a self-reversal spectral feature. Consequently, the observation of the self-reversed spectrum would be a sensitive measure for the purpose of obtaining information on the inhomogeneity in the population-density distribution. In Fig. 3, the depth of the self-reversal, which is defined as a difference between the maximum of the line and the minimum of the dip, is plotted against $\sigma_{1}$. It is seen that both lines of the fine-structure doublet exhibit a similar behavior, i. e., the depth increases with increasing $\sigma_{1}$. In the range of $1.0 \times 10^{-3}<\sigma_{1}<1.5 \times 10^{-3} \mathrm{~m}$, the depth of the self-reversal structure is very sensitive to the value of $\sigma_{1}$, and therefore, the fitting process is expected to be stable. With increasing $\sigma_{1}$ the depth of the self-reversed structure finally saturates toward a certain depth. This saturation depth is essentially determined by a small broadening introduced by the instrumental function. Without the convolution of the instrumental function, the minimum of the self-reversal hole could reach zero. We also performed the fittings with an assumption of $\sigma_{2}=\sigma_{e}=0.002$ or $0.0005 \mathrm{~m}$. The best-fit values of $\sigma_{1}$ were then 0.00304 and $0.00072 \mathrm{~m}$, respectively. The ratio of $\sigma_{1} / \sigma_{2}$ turned out to be always $\sim 1.5$. This seems to be consistent with the understanding that the absorption should balance the emission to give the same observed intensity.

The self-reversal can be understood in terms of the spatial temperature distribution as well, since more population in the lower level means a lower temperature. In Fig. 4, the temperature distribution estimated from the population ratio between the two levels is shown for various values of $\sigma_{1}$, while $\sigma_{2}$ is kept to $1.00 \times 10^{-3} \mathrm{~m}$. In the case of $\sigma_{1}=1.02$ $\times 10^{-3} \mathrm{~m}$, for example, there is virtually no emission and absorption at a distance farther than $2.5 \times 10^{-3} \mathrm{~m}$ from the center, because of the very low population of $\mathrm{Al}$ atoms there. Since the temperature at the position of $2.5 \times 10^{-3} \mathrm{~m}$ from the center is still as high as $3900 \mathrm{~K}$, the temperature difference between the center and the "periphery" is less than 100

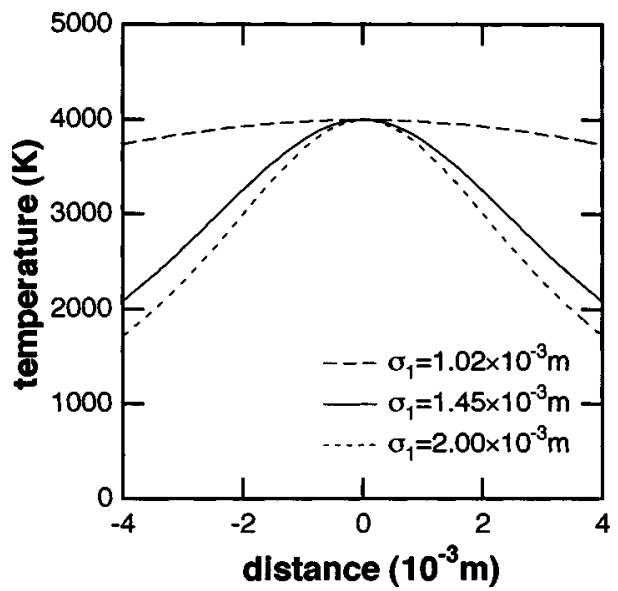

FIG. 4. Spatial distribution of the temperature calculated by the Boltzmann relation from the population ratio between upper and lower levels of the transition. Long broken line, solid line, and short broken line represent $\sigma_{1}$ $=1.02 \times 10^{-3}, 1.45 \times 10^{-3}$, and $2.00 \times 10^{-3} \mathrm{~m}$, respectively, with a fixed $\sigma_{2}$ value of $1.00 \times 10^{-3} \mathrm{~m}$.

$\mathrm{K}$, which is only $2.5 \%$ of the temperature at the center. Nevertheless, our calculation shows a small self-reversal (Fig. 2). This suggests that the self-reversal is very sensitive to the difference in the population distributions of emitters and absorbers, and hence, to the temperature distribution of the light-emitting region.

Concerning the spectral feature in Fig. 1, another point to be noted is that the intensities of the doublet are almost the same, although the radiative rate coefficient of the ${ }^{2} P_{1 / 2}^{\circ}-{ }^{2} S_{1 / 2}(394.4 \mathrm{~nm})$ transition is about a half of that for the ${ }^{2} P_{3 / 2}^{\circ}-{ }^{2} S_{1 / 2}(396.2 \mathrm{~nm})$ transition. ${ }^{35}$ The relative intensity of these two peaks reflects the population densities of the two levels involved in the transition. That is, the optical thickness of the plasma plays an essential role. In Fig. 5, representative spectra calculated with various values of $N_{1 b}$ are shown. In the calculations, all the other parameters, except for $N_{1 a}$ and $N_{20}$ which are determined by the Boltzmann

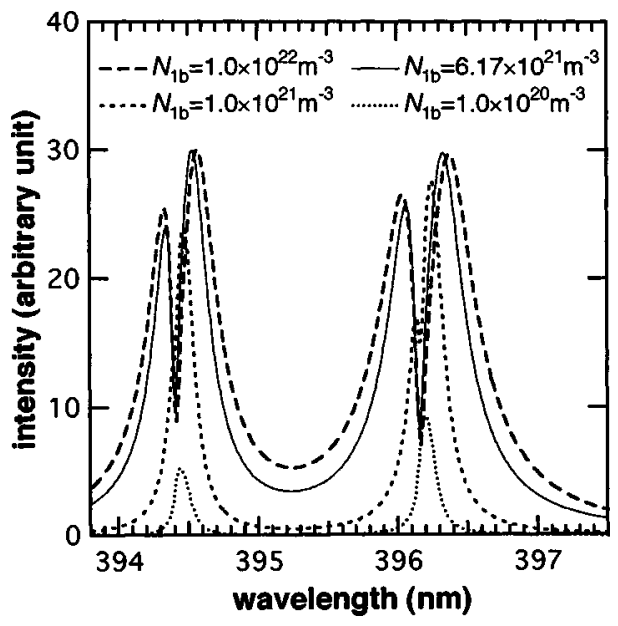

FIG. 5. Representative spectra obtained by the model calculation with various values of $N_{1 b}$. The other parameters, except for $N_{1 a}\left(=2 N_{1 b}\right)$ and $N_{20}$ [calculated by Eq. (16)], were fixed to the values listed in Table I. Long broken line, solid line, short broken line, and dotted line represent $N_{1 b}$ $=1.0 \times 10^{22}, 6.17 \times 10^{21}, 1.0 \times 10^{21}$, and $1.0 \times 10^{20} \mathrm{~m}^{-3}$, respectively. 


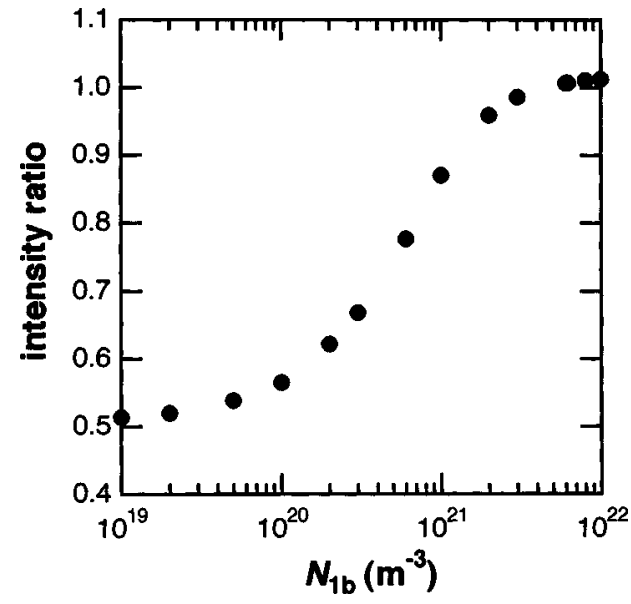

FIG. 6. Peak-intensity ratio of the ${ }^{2} P_{1 / 2}^{\circ}-{ }^{2} S_{1 / 2}$ (394.4 nm) line to the ${ }^{2} P_{3 / 2}^{\circ}-{ }^{2} S_{1 / 2}(396.2 \mathrm{~nm})$ line calculated as a function of $N_{1 b}$. All the other parameters, except for $N_{1 a}\left(=2 N_{1 b}\right)$ and $N_{20}$ [calculated by Eq. (16)], were fixed to the values listed in Table I.

relation, were fixed to the values used in the calculation for Fig. 1 (see Table I). Three features are clearly seen in Fig. 5; (1) the intensities of the doublet are nearly the same and do not increase any more for $N_{1 b}>1.0 \times 10^{21} \mathrm{~m}^{-3}$, (2) the width increases with increasing $N_{1 b}$, and (3) the selfreversal depth decreases with decreasing $N_{1 b}$ and it disappears below $N_{1 b}<1.0 \times 10^{21} \mathrm{~m}^{-3}$. The features (1) and (2) are shown in more detail in Figs. 6 and 7, respectively. In Fig. 6, the ratio of the peak intensity of the $394.4 \mathrm{~nm}$ and $396.2 \mathrm{~nm}$ lines is plotted as a function of $N_{1 b}$. From the radiative rate constant, $A_{2 a}$ and $A_{2 b}$, this ratio is expected to be $\sim 0.5$. The ratio of 0.5 is obtained in the range $N_{1 b}$ $<10^{20} \mathrm{~m}^{-3}$, while it becomes unity for larger $N_{1 b}$ 's. This can be explained in terms of the optical thickness of the central region; with increasing atomic density, and hence, the optical thickness, the emission intensity increases but saturates at the intensity expected from the black body radiation. Since the peaks of the doublet are very close in wavelength,

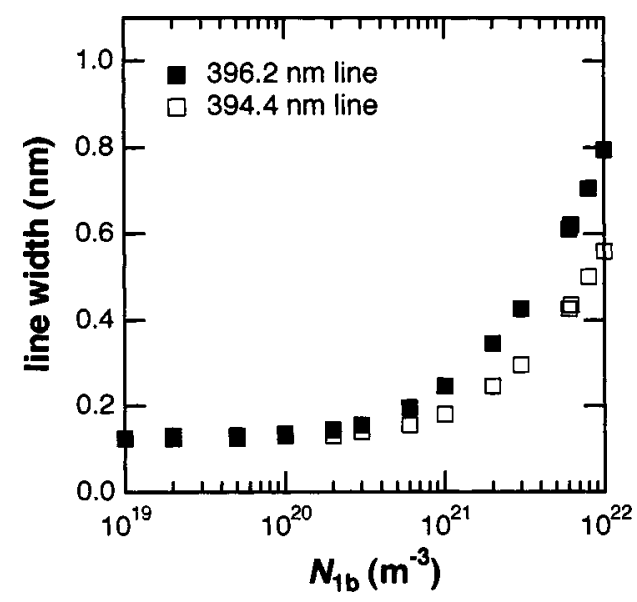

FIG. 7. Linewidth calculated as a function of $N_{1 b}$. Solid squares indicate the width of the ${ }^{2} P_{3 / 2}^{\circ}{ }^{2} S_{1 / 2}(396.2 \mathrm{~nm})$ line while open squares indicate that of the ${ }^{2} P_{1 / 2}^{\circ}{ }^{2} S_{1 / 2}(394.4 \mathrm{~nm})$ line. The parameters other than $N_{1 b}, N_{1 a}$ ( $=2 N_{1 b}$ ) and $N_{20}$ [calculated by Eq. (16)] were fixed to the values listed in Table I.

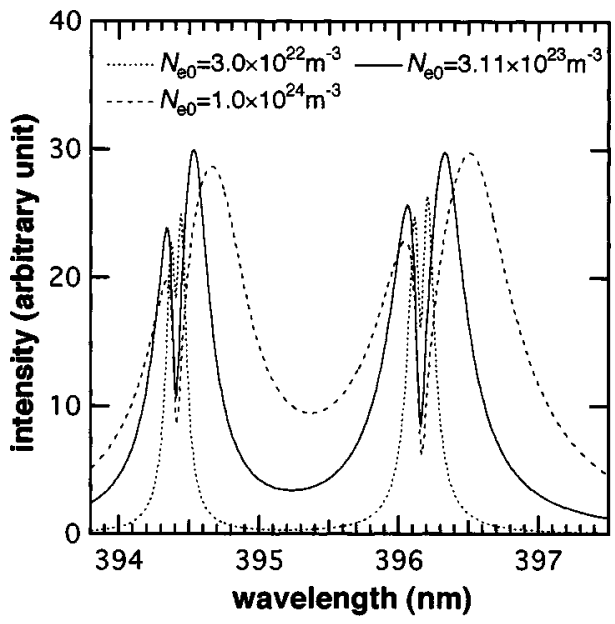

FIG. 8. Representative spectra obtained by the model calculation with various $N_{e 0}$ values. All the other parameters were fixed to the values listed in Table I. Dotted line, solid line, and broken line represent $N_{e 0}=3.0 \times 10^{22}$, $3.11 \times 10^{23}$, and $1.0 \times 10^{24} \mathrm{~m}^{-3}$, respectively.

the intensity expected from the black body radiation is virtually the same for these two lines. As a consequence, the change of the intensity ratio from 0.5 to 1 can be a measure of the variation in the optical thickness, providing information of the population density. In Fig. 7, the variation of the linewidths of the doublet is shown as a function of $N_{1 b}$. The increase of the linewidth is correlated to the intensity ratio of the two peaks. That is, the steep increase of the linewidth starting at $\sim 1 \times 10^{21} \mathrm{~m}^{-3}$ corresponds to the start of the saturation in the intensity ratio given in Fig. 6. As the population of the levels involved in the transition increases, the emission intensity throughout the line profile increases. The intensity at the peak, however, saturates due to the limited intensity of the black body radiation. This saturation at the peak leads to the increase in the linewidth observed here. These results suggest that the population density parameters, such as $N_{1 b}$, can be quite accurately determined through the fitting process.

In Fig. 8, the calculated spectra are presented for various $N_{e 0}$ 's. By varying the values of $N_{e 0}$, the position and the width of the peak drastically change, which originates from the Stark shift and broadening. On the other hand, the position and the width of the self-reversal hole are unchanged. In fact, the width of the self-reversal hole observed in the present measurement was essentially determined by the instrumental resolution. This suggests that the absorption process, which is responsible for the formation of a self-reversal structure, is mainly caused by the atoms in the peripheral region where $N_{e 0}$ is considerably low to give very little Stark effects.

Figures 2, 5, and 8 suggest that the spectra are very sensitive to the adjusting parameters, and therefore, the fittings enable us to obtain accurate values of the parameters, as long as the other parameters have been correctly assumed. The Al spectra have a few important features for the determination of the adjusting parameters: They are the selfreversal depth, the line shift, the width of the envelope of the line, and the intensity ratio of the fine-structure doublet. The 
self-reversal depth is mainly associated with the spatial distribution of the emitters and absorbers, and therefore, helpful for the determination of the parameter, $\sigma_{1}$ with respect to $\sigma_{2}$. The line shift is directly related to the Stark shift and determines the plasma electron density parameter, $N_{e 0}$. On the other hand, the last two features are mainly related to the optical thickness and give us information about the population density, namely $N_{1 b}$ for the present work. The analysis presented in this article confirms that the self-reversed spectra are very useful in determining the spatial distribution of the population density for the species in the laser ablation plasma.

\section{CONCLUSION}

In conclusion, we have developed a model, which is based on the radiative transfer of the atomic emission, to account for the self-reversed line spectra observed in the laser ablation plume. After a general description of the model, we have specifically applied it for the analysis of the laserablated Al plasma in air. In the model, the effects of the position-dependent Stark shift and broadening were taken into account, and the plasma electron density, populations of the atomic levels involved in the transition, and their spatial distribution were considered to be the fitting parameters. Our model successfully reproduced the spectra of the finestructure doublet from $\mathrm{Al}$ atoms in the ablation plume.

Our systematic analysis presented in this work has confirmed that the spatial distribution of the plasma parameters and atomic populations, which are very difficult to determine otherwise, can be accurately estimated by fitting the parameters in the model to the experimental self-reversed spectra. Finally, we would like to note that the present method is applicable to various kinds of emitting species in a wide range of delay time. It will contribute to the analysis of dynamical aspects of laser-produced plasma from various target materials.

\section{ACKNOWLEDGMENTS}

The authors would like to acknowledge Professor Mahito Mabuchi for stimulating discussions. This work is financially supported by a Grant-in-Aid for scientific research from the Japan Society for the Promotion of Science.

\footnotetext{
${ }^{1}$ Laser Ablation and Desorption, edited by J. C. Miller and R. F. Haglund, Jr. (Academic, New York, 1998).

${ }^{2}$ Proceedings of the 6th International Conference on Laser Ablation, edited by K. Murakami et al., Tsukuba, Japan, October, 2001

${ }_{3}^{3}$ Appl. Phys. A: Mater. Sci. Process. 69, (1999).
}

${ }^{4}$ V. Majidi and M. R. Joseph, Crit. Rev. Anal. Chem. 23, 143 (1992).

${ }^{5}$ K. Y. Song, I. Lee, and J. Sneddon, Appl. Spectrosc. Rev. 32, 183 (1997).

${ }^{6}$ D. B. Geohegan and A. A. Puretzky, Appl. Phys. Lett. 67, 197 (1995).

${ }^{7}$ P. Engst, P. Kubát, P. Boháček, and J. Wild, Appl. Phys. Lett. 64, 2025 (1994).

${ }^{8}$ H. Wang, A. P. Salzberg, and B. R. Weiner, Appl. Phys. Lett. 59, 935 (1991).

${ }^{9}$ R. D. Cowan and G. H. Dieke, Rev. Mod. Phys. 20, 418 (1948)

${ }^{10} \mathrm{H}$. Zwicker, in Plasma Diagnostics, edited by W. Lochte-Holtgreven (North-Holland, Amsterdam, 1968), p. 214.

${ }^{11}$ A. Skudra and G. Revalde, J. Quant. Spectrosc. Radiat. Transf. 61, 717 (1999).

${ }^{12}$ J. J. de Groot and A. G. Jack, J. Quant. Spectrosc. Radiat. Transf. 13, 615 (1973).

${ }^{13}$ H. P. Stormberg and R. Schäfer, J. Quant. Spectrosc. Radiat. Transf. 33, 27 (1985).

${ }^{14}$ B. Yaakobi, D. Steel, E. Thorsos, A. Hauer, B. Perry, S. Skupsky, J. Geiger, C. M. Lee, S. Letzring, J. Rizzo, T. Mukaiyama, E. Lazarus, G. Halpern, H. Deckman, J. Delettrez, J. Soures, and R. McCrory, Phys. Rev. A 19, 1247 (1979).

${ }^{15}$ J. D. Kilkenny and R. W. Lee, Phys. Rev. A 22, 2746 (1980).

${ }^{16}$ N. D. Delamater, C. F. Hooper, Jr, R. F. Joyce, L. A. Woltz, N. M. Ceglio, R. L. Kauffman, and R. W. Lee, Phys. Rev. A 31, 2460 (1985).

${ }^{17}$ S. Sorge, A. Wierling, G. Röpke, W. Theobald, R. Sauerbrey, and T. Wilhein, J. Phys. B 33, 2983 (2000).

${ }^{18}$ H. A. Phyllips, H. L. Lancaster, M. B. Denton, K. Rozsa, and P. Apai, Appl. Spectrosc. 42, 572 (1988).

${ }^{19}$ E. K. Kemsley and P. S. Belton, Spectrochim. Acta, Part A 51, 371 (1995).

${ }^{20}$ J. Hermann, C. Boulmer-Leborgne, and D. Hong, J. Appl. Phys. 83, 691 (1998).

${ }^{21}$ R. F. G. Meulenbroeks, P. A. A. van der Heijden, M. C. M. van de Sanden, and D. C. Schram, J. Appl. Phys. 75, 2775 (1994).

${ }^{22}$ B. Toftmann, J. Schou, T. N. Hansen, and J. G. Lunney, Phys. Rev. Lett. 84, 3998 (2000)

${ }^{23}$ F. J. Gordillo-Vázquez, A. Perea, J. A. Chaos, J. Gonzalo, and C. N. Afonso, Appl. Phys. Lett. 78, 7 (2001).

${ }^{24}$ H. R. Griem, Principles of Plasma Spectroscopy (Cambridge University Press, Cambridge, UK, 1997).

${ }^{25}$ R. Loudon, The Quantum Theory of Light, 2nd ed. (Oxford Science, Oxford, 1983).

${ }^{26}$ H. R. Griem, Plasma Spectroscopy (McGraw-Hill, New York, 1964).

${ }^{27}$ G. Bekefi, C. Deutsch, and B. Yaakobi, in Principles of Laser Plasmas, edited by G. Bekefi (Wiley, New York, 1976), p. 549.

${ }^{28}$ N. Konjevic and M. S. Dimitrijevic, J. Phys. Chem. Ref. Data 13, 619 (1984).

${ }^{29}$ K. Saito, K. Takatani, T. Sakka, and Y. H. Ogata, Appl. Surf. Sci (in press).

${ }^{30}$ T. Sakka, S. Iwanaga, Y. H. Ogata, A. Matsunawa, and T. Takemoto, J. Chem. Phys. 112, 8645 (2000).

${ }^{31}$ Y. F. Lu, Z. B. Tao, and M. H. Hong, Jpn. J. Appl. Phys., Part 1 38, 2958 (1999).

${ }^{32}$ R. W. Dreyfus, R. Kelly, and R. E. Walkup, Appl. Phys. Lett. 49, 1478 (1986).

${ }^{33}$ G. Mehlman, D. B. Chrisey, P. G. Burkhalter, J. S. Horwitz, and D. A. Newman, J. Appl. Phys. 74, 53 (1993).

${ }^{34}$ P. S. P. Wei, J. Appl. Phys. 48, 4196 (1977).

${ }^{35}$ W. L. Wiese, M. W. Smith, and B. M. Glennon, Atomic Transition Probabilities, National Standard Reference Data System (National Bureau of Standards, Washington DC, 1966).

${ }^{36}$ X. T. Wang, B. Y. Man, G. T. Wang, Z. Zhao, Y. Liao, B. Z. Xu, Y. Y. Xia, L. M. Mei, and X. Y. Hu, J. Appl. Phys. 80, 1783 (1996). 